Jonathan Cavanagh

Reflections on Class Theory suggested by Analysis of the Peruvian Military Regime, 1968-1979.

(zu beziehen durch: B. Fries, Laboer Weg 62 A, D-2305 Heikendorf), Göttingen 1980, 558

+ IX S., Bundesrepublik: 60,- DM, USA: $34.80 \$$

Die vorliegende Göttinger Dissertation, die im Rahmen der Arbeit einer Forschungsgruppe über Peru an der Abteilung für internationale Beziehungen der Göttinger Universität ${ }^{1}$ entstanden ist, verfolgt gleichzeitig zwei Ziele: das peruanische Militärregime unter Velasco herrschaftssoziologisch zu verorten und die Marx'sche (also nicht marxistische) Klassentheorie als sozialwissenschaftlichen Beitrag zu werten. Die zentrale These von Cavanagh, die sowohl in Abgrenzung zu schematisch verfahrenden Marxisten als auch zu den Gegnern der Marx'schen Klassentheorie entwickelt wird, lautet, daß diese Theorie eine Herrschaftskritik darstellt. Cavanagh weist die Interpretation der Marx'schen Klassentheorie als ein ,,fertiges“ (ready-made) Schema oder als Instrumentarium für die behaviouristische Analyse konkret-historischer Situationen teilweise zurück; für ihn ist sie substantiell eine Kritik bestehender Produktionsverhältnisse, die auch in konkret-historischen Situationen also nichts an ihrer ,,Wahrheit" verbüßt hat. Um diese These zu untermauern, baut er in seine Arbeit eine gründliche Interpretation des ,,Kapitals“ und eine auf einer neuen Lektüre basierende Deutung des ,Achtzehnten Brumaires“.

Cavanaghs Arbeit hat folgenden Aufbau: Die beiden ersten Kapitel enthalten eine auf den neuesten Forschungsergebnissen (bes. auf Fitzgerald und Thorp) basierende Einführung in den im Mittelpunkt dieser Arbeit stehenden Gegenstand, in das peruanische Militärregime unter Velasco. Das dritte Kapitel ist der Auseinandersetzung mit den in Peru entstandenen Arbeiten zur klassenanalytischen Deutung des Velasco-Regimes gewidmet. Das vierte Kapitel vermittelt eine auf einem hohen Niveau geführte theoretische Diskussion über die Marx'sche Klassenanalyse und zugleich einen Exkurs über modernisierungstheoretisch inspirierte Alternativen zu dieser Analyse (Huntington, Nordlinger u. a.). Im fünften Kap. geht Cavanagh auf die zweite Phase des peruanischen Militärregimes (seit 1975) ein und versucht einen eigenen historiographischen Ansatz zu entwickeln.

Das dritte und das vierte Kapitel stehen im Mittelpunkt der Cavanaghschen Dissertation und enthalten ihren originellen Beitrag. Die ideologiekritischen Analysen des dritten Kapitels verdeutlichen, daß die in Peru verbreiteten klassentheoretischen Analysen über das Velasco-Militärregime auf ökonomischem Reduktionismus beruhen, subjektivistisch sind und eine ahistorische schematische Anwendung der Marx'schen Klassentheorie vorweisen. Die Marx'sche Kritik beruht nach Cavanagh nicht auf einer moralischen Wertung bzw. auf einer dogmatischen oder subjektivistischen Einschätzung bestehender gesellschaftlicher Verhältnisse. Sie ist eine Kritik der gegebenen kapitalistischen Form der sozialen Organisation der Produktion, die in Verbindung mit einer stringenten ,,economic constraints analysis“ ihren vollen Wert erreicht. Der Verfasser untermauert diese These durch seine gründliche Exegese des ,,Kapitals“ und des ,,Achtzehnten Brumaires“ im vierten Kapitel, wobei er die scholastische Manier des Marxisierens, die man in der marxistisch inspirierten Literatur nicht selten vorfindet, ablehnt, also auch die Grenzen der Marx-Exegese deutlich überschreitet und die Marx'sche Klassenanalyse streng im Hinblick auf ihre sozialwissenschaftliche Aussagefähigkeit, d. h. ihren möglichen Beitrag zur Deutung konkret politischer Verhältnisse überprüft.

1 Vgl. meine Rezension der Arbeit von A. Möller, Städtische Marginalität und Militärregierung in Peru, 1977, in VRU 12 (1979), S. 290-292. 
Sehr interessant ist die in der vorliegenden Arbeit geführte Diskussion über die Möglichkeit des Einbaus einer Handlungstheorie in die Marx’sche Klassenanalyse. In seinem „,Postscript" diskutiert Cavanagh im Licht der über Peru geleisteten Analysen einen Versuch des Frankfurter Soziologen Gerhard Brandt, die Sozialstrukturanalyse mit einer Handlungstheorie zu verbinden. Cavanagh teilt mit Brandt die Einsicht, daß eine Klassenanalyse auch eine handlungstheoretische Deutungskapazität vorweisen muß, gibt aber zu bedenken, wie problematisch es ist, die Handlungstheorie in die Marx'sche Klassenanalyse zu integrieren. Denn die letztere ist ja eine Kritik, während die Handlungstheorie auf einem Instrumentarium beruht. Auch Brandt überwindet diese Schwierigkeit nicht und bleibt, wie Cavanagh mit Recht sagt, bei einem Plädoyer.

Die Dissertation von Cavanagh öffnet einerseits neue Perspektiven für eine neue Marx-Rezeption und enthält ein Plädoyer für eine ,,re-lecture“ der Marx'schen Schriften im Hinblick auf die bereits geleisteten Interpretationen über den Stellenwert des Spätwerkes, d. h. dafür, daß der Klassenbegriff neu durchdacht werden müßte. Andererseits bietet die Cavanaghsche Arbeit eine erstmalige Auswertung von peruanischen Primärquellen. Cavanagh war durch mehrere längere Peru-Aufenthalte in der Lage, spanischsprachige Quellen, die peruanische „,Klassenanalysen“ enthalten, zu erschließen. Seine Interpretation dieser Schrift belegt, daß der peruanische Marxismus kein hohes theoretisches Niveau vorweisen kann und eher auf Glaubensbekenntnissen als auf Analysen basiert.

Die Göttinger Dissertation von Cavanagh ist ein exzellenter sozialwissenschaftlicher Beitrag für die entwicklungstheoretische Diskussion und eine Bereicherung der theorie-orientierten Literatur über Peripherie-Gesellschaften. Cavanagh legt eine gründliche Peru-Monographie vor, die allerdings nicht nur für Peru-Experten geschrieben ist, sondern zu Recht beansprucht, einen theoriebildenden Beitrag zu vermitteln.

Bassam Tibi

\section{GISELHER FOETH}

\section{Investitionen in Lateinamerika.}

Internationale Verträge und nationale Bestimmungen.

Bearbeitet von Giselher Foeth. Hrsgg. von Gottfried Zieger.

Alfred Metzner Verlag GmbH, Frankfurt am Main, 1979, 609 S., DM 95,-(Bd. 45 der Reihe

DOKUMENTE, hrsgg. vom Institut für Internationale Angelegenheiten der Universität Hamburg, vom Institut für Internationales Recht an der Universität Kiel und vom Institut für Völkerrecht der Universität Göttingen).

Nach wie vor ist Lateinamerika eines der Hauptziele deutscher Kapitalanlagen im Ausland. Allein Brasilien nimmt heute mit 4,5 Mrd. hinter den USA, Belgien/Luxemburg, Frankreich und der Schweiz den fünften Platz im Weltvergleich aller deutschen Auslandsinvestitionen ein, die sich zwischen 1952 und 1979 auf insgesamt 58,18 Mrd. DM summiert haben. Von den 28,5\%, die hiervon in die Dritte Welt flossen, beträgt der Anteil Lateinamerikas allein fast die Hälfte (47\%). Deutsche Weltfirmen wie etwa Hoechst oder BASF erzielten z. B. 1978 in Lateinamerika Umsätze in Höhe von 6 \% bzw. 10,2 \% ihres Weltumsatzes, wobei die Umsatzraten aus lokaler Produktion im Durchschnitt etwa zwei Drittel ausmachten. Angesichts solcher Zahlen bedarf ein Werk wie das hier anzuzeigende keiner weiteren Legitimation. Der Autor ist überdies als ehemaliger wissenschaftlicher Referent am Institut für Völkerrecht an der Göttinger Universität und kraft seiner Erfahrungen im internationalen Finanzierungsgeschäft einer deutschen Großbank für eine solche Aufgabe bestens ausgewiesen. 